\title{
Measuring the Mass Distribution in Galaxy Clusters
}

\author{
Margaret J. Geller \\ Antonaldo Diaferio \\ Kenneth J. Rines \\ Western Washington University, ken.rines@wwu.edu \\ Ana Laura Serra
}

Follow this and additional works at: https://cedar.wwu.edu/physicsastronomy_facpubs

Part of the Astrophysics and Astronomy Commons

\section{Recommended Citation}

Geller, Margaret J.; Diaferio, Antonaldo; Rines, Kenneth J.; and Serra, Ana Laura, "Measuring the Mass Distribution in Galaxy Clusters" (2013). Physics \& Astronomy. 35.

https://cedar.wwu.edu/physicsastronomy_facpubs/35 


\title{
MEASURING THE MASS DISTRIBUTION IN GALAXY CLUSTERS
}

\author{
Margaret J. Geller $^{1}$, Antonaldo Diaferio ${ }^{2,5}$, Kenneth J. Rines ${ }^{3}$, and Ana Laura Serra ${ }^{4,5,6}$ \\ ${ }^{1}$ Smithsonian Astrophysical Observatory, 60 Garden St., Cambridge, MA 02138, USA; mgeller@ cfa.harvard.edu \\ 2 Dipartimento di Fisica, Università degli Studi di Torino, via P. Giuria 1, I-10125 Torino, Italy; diaferio@ @h.unito.it \\ ${ }^{3}$ Department of Physics \& Astronomy, Western Washington University, Bellingham, WA 98225, USA; kenneth.rines@wwu.edu \\ ${ }^{4}$ INAF, Osservatorio Astronomico di Torino, via Osservatorio 20, I-10025 Pino Torinese (TO), Italy; serra@ @o.infn.it \\ ${ }^{5}$ Dipartimento di Fisica, Università degli Studi di Torino, via P. Giuria 1, I-10125 Torino, Italy \\ ${ }^{6}$ INFN, Sezione di Torino, via P. Giuria 1, I-10125 Torino, Italy \\ Received 2012 September 19; accepted 2012 November 9; published 2013 January 28
}

\begin{abstract}
Cluster mass profiles are tests of models of structure formation. Only two current observational methods of determining the mass profile, gravitational lensing, and the caustic technique are independent of the assumption of dynamical equilibrium. Both techniques enable the determination of the extended mass profile at radii beyond the virial radius. For 19 clusters, we compare the mass profile based on the caustic technique with weak lensing measurements taken from the literature. This comparison offers a test of systematic issues in both techniques. Around the virial radius, the two methods of mass estimation agree to within $\sim 30 \%$, consistent with the expected errors in the individual techniques. At small radii, the caustic technique overestimates the mass as expected from numerical simulations. The ratio between the lensing profile and the caustic mass profile at these radii suggests that the weak lensing profiles are a good representation of the true mass profile. At radii larger than the virial radius, the extrapolated Navarro, Frenk \& White fit to the lensing mass profile exceeds the caustic mass profile. Contamination of the lensing profile by unrelated structures within the lensing kernel may be an issue in some cases; we highlight the clusters MS0906+11 and A750, superposed along the line of sight, to illustrate the potential seriousness of contamination of the weak lensing signal by these unrelated structures.
\end{abstract}

Key words: cosmology: observations - dark matter - galaxies: clusters: individual (MS0906+11, A750) galaxies: kinematics and dynamics

Online-only material: color figure

\section{INTRODUCTION}

Mass profiles of clusters of galaxies are tests of models of structure formation on scales from $40 h^{-1} \mathrm{kpc}$ to $5 h^{-1} \mathrm{Mpc}$. They probe the nature of dark matter and enable exploration of the link between cosmology and cluster formation.

Navarro et al. (1997, NFW) spurred interest in cluster mass profiles when they demonstrated that the profile for virialized halos has a universal form independent of the initial power spectrum of density fluctuations and of the cosmological parameters. Two parameters a mass (within a radius surrounding a region with a specified average density contrast) and a central concentration completely define the NFW profile. NFW predicted greater concentration for lower mass systems reflecting their earlier formation time. More recently, Merritt et al. (2006), Navarro et al. (2010), and others have argued that the shapes of dark matter halo mass profiles are better approximated by the three-parameter Einasto profile (1965; see Coe 2010 for a tutorial comparison of the profiles). The NFW form remains a good approximation; it is thus widely applied to characterize observations.

Combined strong and weak lensing observations of several massive clusters suggest a tension between observed cluster mass profiles and theoretical predictions. A set of eight wellstudied clusters with masses $\gtrsim 5 \times 10^{14} h^{-1} M_{\odot}$ are more centrally concentrated than models predict (Broadhurst et al. 2008; Oguri et al. 2009; Sereno et al. 2010; Postman et al. 2012). The observations are impressive because they probe the central region directly and the resulting mass distribution is independent of the detailed dynamical state of the cluster. These

\footnotetext{
7 INFN, Sezione di Torino, via P. Giuria 1, I-10125 Torino, Italy
}

results suggest an earlier formation time for massive clusters than predicted by the standard model (Duffy et al. 2008; Prada et al. 2012). On the other hand, the presence of strong lensing arcs may select for more centrally concentrated clusters than those typical of the mass range (Hennawi et al. 2007; Oguri \& Blandford 2009; Meneghetti et al. 2010).

Umetsu et al. (2011) combine four clusters with a characteristic mass of $\sim 1.5 \times 10^{15} h^{-1} M_{\odot}$ to derive a single high-precision mass profile covering the range $40 h^{-1} \mathrm{kpc}$ to $2.8 h^{-1} \mathrm{Mpc}$. The profile agrees impressively well with the NFW prediction even at radii exceeding the virial radius. The concentration of this summed profile exceeds typical model predictions. Okabe et al. (2010) derive stacked weak lensing profiles for two larger sets of less massive clusters; the profiles are less concentrated than the Umetsu et al. (2011) profile, suggesting that the smaller Umetsu et al. (2011) sample may be biased. Like the Umetsu et al. (2011) profile, the Okabe et al. (2010) profiles are a superb match to the NFW form over a large radial range.

In addition to comparisons with theoretical expectations, weak lensing masses and mass profiles have been tested against other observational approaches. Masses derived from X-ray observations and from equilibrium dynamical analyses of cluster redshift surveys are generally in impressive agreement with the lensing results (e.g., Irgens et al. 2002; Diaferio et al. 2005; Hoekstra 2007; Okabe et al. 2010). A drawback of these comparisons is that most other observational techniques, unlike lensing, assume dynamical equilibrium and they do not extend to large radius.

Here we compare a set of weak lensing mass profiles from the literature (Hoekstra 2007; Okabe \& Umetsu 2008; Lemze et al. 2008; Umetsu et al. 2009; Okabe et al. 2010; Oguri et al. 2010) with profiles determined from the caustic technique. 
Diaferio and Geller (1997, DG97) and Diaferio (1999, D99) used simulations to determine the cluster mass distribution without requiring the equilibrium assumption. They showed that the amplitude of the trumpet-shaped pattern typical of clusters in redshift space (Kaiser 1987; Regös \& Geller 1989) is a measure of the escape velocity from the cluster. The mass estimator based on this identification enables measurement of the cluster mass profile within the virialized central region and throughout the surrounding infall region. Rines et al. (2012) show that the NFW profile is a good representation of the data on scales $\lesssim 2 h^{-1} \mathrm{Mpc}$; on larger scales the profiles appear to steepen.

Applications of the caustic technique include the analysis of a sample of nearby clusters (Rines et al. 2003), a sample of 72 clusters included in the Sloan Digital Sky Survey (SDSS; Rines \& Diaferio 2006; CIRS), a sample of X-ray-selected groups (Rines \& Diaferio 2010), and detailed studies of individual systems (e.g., Geller et al. 1999; Reisenegger et al. 2000; Drinkwater et al. 2001; Biviano \& Girardi 2003; Lemze et al. 2009; Lu et al. 2010). These studies include comparisons with masses determined from the X-ray and from Jeans analysis; in general the masses agree reasonably well. As for the weak lensing profiles, caustic mass profiles extending beyond the virial radius are consistent with the NFW form over a wide range of scales (Geller et al. 1999; Rines \& Diaferio 2006; Rines et al. 2012). In contrast with the smaller sample of clusters studies with weak lensing, kinematic analysis of these large, complete samples of X-ray-selected clusters imply concentrations consistent with theory (Rines \& Diaferio 2006). These results underscore the importance of sample selection in the determination of mass profiles (see, e.g., Postman et al. 2012).

Comparison of kinematic mass profiles with weak lensing offers an observational test of both techniques. Ultimately, if the systematic biases in these techniques can be understood, this kind of comparison could be a route to testing alternative theories of gravity (Lam et al. 2012) and the dark matter equation of state (e.g., Faber \& Visser 2006; Serra \& Domínguez Romero 2011).

The number of clusters with weak lensing observations and with the dense spectroscopy necessary for application of the caustic technique has been small. Lemze et al. (2009) compare the caustic mass profiles with the weak lensing profiles for A1689 and find impressive agreement. Umetsu et al. (2010) make a similar detailed comparison for $\mathrm{Cl} 10024+1654$ and show that the weak lensing mass significantly exceeds the caustic mass at radii approaching the virial radius. The caustic mass is consistent with earlier weak lensing estimates (Kneib et al. 2003) that measure the mass distribution only in the central halo of this complex system. Diaferio et al. (2005) used earlier data for three clusters, A2390, MS1358.4+6245, and C10024+1654 to show that the profiles are in reasonable agreement.

The HeCS (Hectospec Cluster Survey; Rines et al. 2012) sample of clusters in the redshift range $0.1<z<0.3$ substantially increases the overlap between the set of clusters with extensive spectroscopy and those with weak lensing measurements. Among the 58 clusters in the X-ray flux-limited HeCS sample, 17 have published weak lensing mass profiles. Two additional clusters in the CIRS (Cluster Infall Regions in SDSS) cluster sample drawn from SDSS also have weak lensing profiles. Here we compare the caustic mass profile estimates for these 19 systems with the weak lensing estimates.

We review the HeCS and CIRS samples in Section 2. In Section 3 we briefly review the caustic technique we apply. We compare the caustic mass profiles with published weak lensing mass profiles and examine possible sources of disagreement. We discuss the results in Section 4 and conclude in Section 5.

\section{CLUSTER SURVEYS AND WEAK LENSING MASS PROFILES}

CIRS (Rines \& Diaferio 2008) and HeCS (Rines et al. 2012) are redshift surveys of X-ray flux-limited samples of clusters of galaxies. There are typically more than $\sim 150$ spectroscopically confirmed cluster members inside the turnaround radius. Together these samples include 130 clusters. The CIRS clusters are typically at $z \lesssim 0.1$; the $\mathrm{HeCS}$ clusters are in the range $0.1<z<0.3$. Here we briefly review the cluster redshift surveys and sources of the weak lensing profiles we compare with our dynamical estimates.

\subsection{The Cluster Redshift Surveys}

Rines \& Diaferio (2008) extracted the CIRS sample from the Fourth Data Release of the SDSS (Adelman-McCarthy et al. 2006). The cluster sample is X-ray flux limited with $f_{x}>3 \times 10^{-12} \mathrm{erg} \mathrm{s}^{-1} \mathrm{~cm}^{-2}(0.1-2.4 \mathrm{keV})$. The clusters have redshift $z<0.1$ and thus the SDSS redshift survey reaches to approximately $M_{r, 0.1}^{*}+1$ within each cluster. This photometric limit guarantees a large enough sample of cluster members to determine the cluster boundaries in redshift space. There are 72 clusters in the sample and the clusters contain a total of more than 15,000 members. Approximately a third of the members are projected within the virial radius; the rest are projected outside the virial radius but within the infall region.

Rines et al. (2012) enlarge the number of spectroscopically well-sampled clusters by using the 300 fiber Hectospec on the $6.5 \mathrm{~m}$ MMT to measure redshifts in $58 \mathrm{X}$-ray-selected clusters. These clusters span the redshift range $0.1<z<0.3$. The X-ray flux limit is $5 \times 10^{-12} \mathrm{erg} \mathrm{s}^{-1} \mathrm{~cm}^{-2}$ in the ROSAT band (Ebeling et al. 1998; Böhringer et al. 2000).

To maximize the efficiency of the Hectospec observations, targets for spectroscopic observations are within \pm 0.3 mag of the red sequence at the cluster redshift. This strategy typically yielded $~ 150-200$ members in each cluster with two pointings of the 300 fiber Hectospec (Fabricant et al. 2005) per cluster. The HeCS survey includes more than 20,000 new redshifts; among these more than 10,000 are cluster members.

For all 130 clusters in the CIRS and HeCS samples, the redshift survey is dense enough to define the boundaries of the cluster in redshift space. We have applied the caustic technique uniformly to each set of clusters. Here we focus on the subset of 19 clusters in the two surveys that have mass profiles derived from weak lensing observations.

\subsection{Weak Lensing}

We searched the literature for weak lensing mass profiles for clusters in both the CIRS and HeCS samples. We found 19 matches; 2 in CIRS and 17 in HeCS. Table 1 lists the clusters and the source of the weak lensing profile.

Two clusters in the CIRS sample have weak lensing mass profiles derived from Subaru data (Okabe \& Umetsu 2008; Umetsu et al. 2009). Okabe \& Umetsu (2008) derived mass profiles for seven merging clusters; three of these systems are in the HeCS survey (see Table 1) and one (A1750) is in the CIRS sample. Umetsu et al. (2009) also studied the complex cold front system A2142 (in CIRS). The X-ray properties of these clusters suggest that their central regions are not in dynamical equilibrium. 
Table 1

Weak Lensing Observations of HeCS and CIRS Clusters

\begin{tabular}{lll}
\hline \hline Cluster Name & Telescope & \multicolumn{1}{c}{ Reference } \\
\hline A267 & Subaru & Okabe et al. (2010) \\
A689 & CFHT & Hoekstra (2007) \\
A697 & Subaru & Oguri et al. (2010) \\
MS0906 & Subaru & Okabe et al. (2010) \\
A963 & CFHT & Hoekstra (2007) \\
& Subaru & Okabe et al. (2010) \\
A1689 & CFHT & Hoekstra (2007) \\
& CFHT & Hoekstra (2007) \\
A1750 ${ }^{\text {a,b }}$ & HST & Lemze et al. (2008) \\
A1758 & Subaru & Okabe \& Umetsu (2008) \\
A1763 & Subaru & Okabe \& Umetsu (2008) \\
A1835 & CFHT & Hoekstra (2007) \\
A1914 & Subaru & Okabe et al. (2010) \\
A2009 & Subaru & Okabe \& Umetsu (2008) \\
A2034 & Subaru & Okabe et al. (2010) \\
A2142 a,b & Subaru & Okabe \& Umetsu (2008) \\
A2219 & Subaru & Umetsu et al. (2009) \\
RXJ1720 & Subaru & Okabe et al. (2010) \\
A2261 & CFHT & Hoekstra (2007) \\
A2631 & Subaru & Okabe et al. (2010) \\
RXJ2129 & Subaru & Okabe et al. (2010) \\
\hline
\end{tabular}

Notes.

${ }^{a}$ Denotes a cluster selected for weak lensing observation as merging or cold front system.

b Denotes a cluster in the CIRS sample. All other systems are in the HeCS sample.

Among the 17 clusters in $\mathrm{HeCS}$ with weak lensing mass profiles, 15 are also derived from Subaru observations (Okabe \& Umetsu 2008; Okabe et al. 2010; Umetsu et al. 2009). Six clusters have weak lensing profiles derived from Canada-France-Hawaii Telescope (CFHT) observations (Hoekstra 2007); among these, three are clusters that also have Subaru weak lensing profiles. We use the differences in the Subaru- and CFHT-derived profiles as a measure of the uncertainty in the lensing profile. For one cluster, A1689, there is a CFHT-derived profile (Hoekstra 2007) and a profile derived from Hubble Space Telescope (HST) data (Lemze et al. 2008). The clusters A1763 and MS0906+11 have profiles derived from CFHT data alone (Hoekstra 2007). In cases where there is more than one profile derived from Subaru observations, we chose the most recent analysis. Table 1 summarizes the sources of the weak lensing profiles.

Figure 1 shows the distribution of masses for HeCS clusters (determined from the caustic technique; Rines et al. 2012) within the radius containing an average density 200 times the critical density, $M_{200}^{\text {caus }}$ (thin histogram). The heavy histogram shows the distribution for the subset with weak lensing profiles in the literature. Clearly the systems with weak lensing measurements tend to be more massive. This shift toward more massive clusters in the lensing sample results from selection. The HeCS clusters are a flux-limited sample; the clusters with weak lensing profiles are generally among the most intrinsically luminous (and thus generally most massive) X-ray clusters.

For the sample of $17 \mathrm{HeCS}$ clusters, the 15 Subaru profiles were analyzed by collaborating groups of investigators who use consistent techniques; these profiles thus provide a reasonably uniform testbed for the caustic profiles we derive.

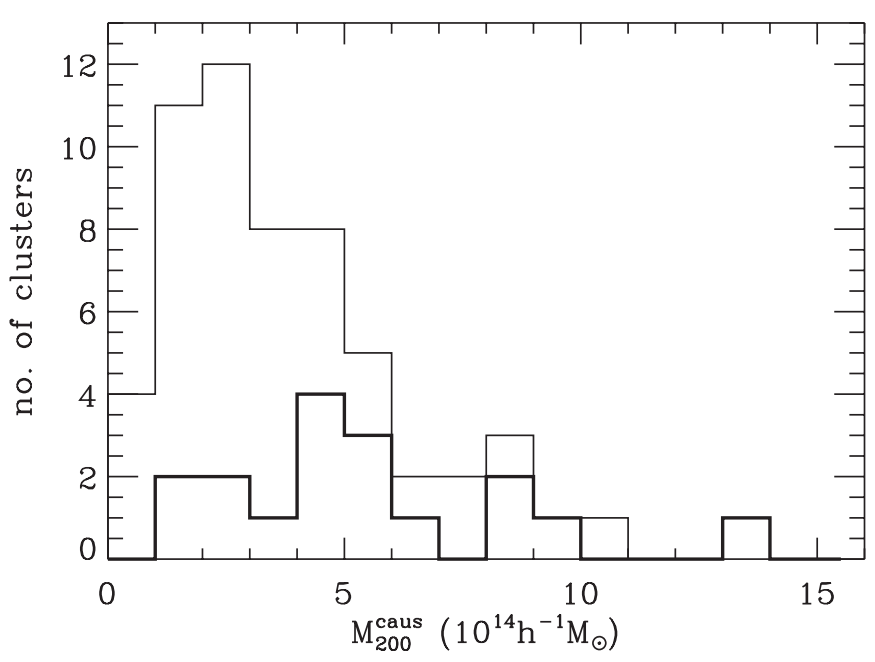

Figure 1. Distribution of caustic masses, $M_{200}^{\text {caus }}$ for the HeCS sample (thin line) and for the subsample with weak lensing mass profiles (heavy line). The observed lensing clusters tend to be more massive.

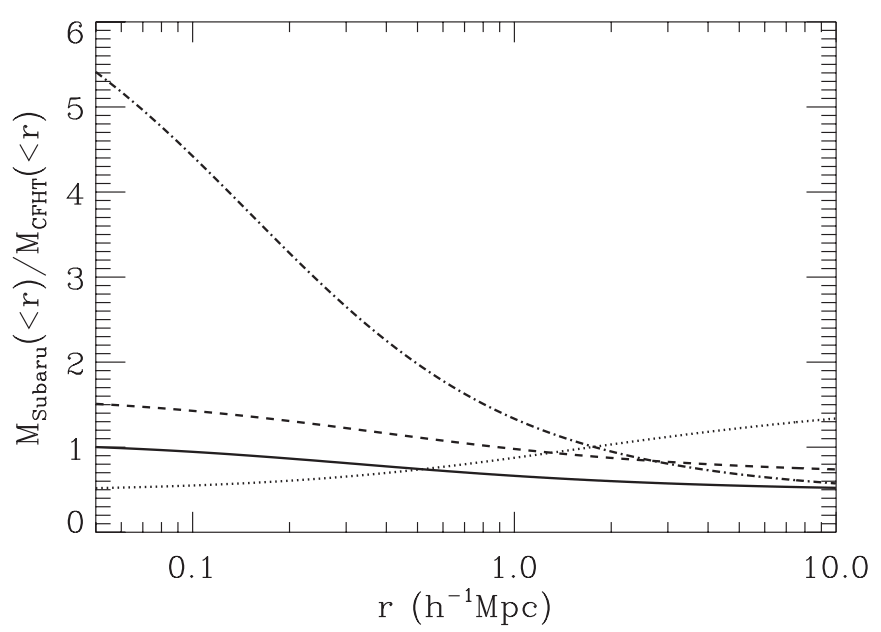

Figure 2. Ratio of weak lensing profiles for clusters observed with more than one facility. We plot $M_{\text {Subaru }} / M_{\text {CFHT }}$ for all cases except A1689 where we plot $M_{H S T} / M_{\text {Subaru }}$. We plot profile ratios for A1689 (dot-dashed curve), A2219 (dashed), A267 (solid), and A963 (dotted). These ratios suggest that systematic errors in the lensing profiles are $\sim 50 \%$.

Figure 2 shows the ratio between the two weak lensing profiles for each of the four clusters observed with Subaru and CFHT or with CFHT and HST. The figure shows that for groundbased facilities the profiles differ by $\lesssim 50 \%$ throughout the radial range. The profile derived from $H S T$ data is much more centrally concentrated than the ground-based profile for A1689.

Systematic errors in weak lensing mass profiles may originate from both astrophysical and data reduction issues. We cannot say which, if any, of these issues affect the relative profiles in Figure 2, but we review the possibilities. The three issues that appear to produce the largest systematic effects are astrophysical (Okabe et al. 2010; Oguri et al. 2010): contamination of the source population by faint galaxies in the cluster, errors in the source redshift, and projection of foreground/background structures.

Dilution of the source catalog by faint cluster members is a potential systematic with an obviously decreasing impact as a function of distance from the cluster center. The catalogs of Okabe et al. (2010) for the clusters A963 and A2009 probably suffer from this dilution effect because the authors used imaging 
data in a single band and thus could not discriminate between member and background galaxies. At small radii where the contamination is potentially largest, this kind of dilution leads to an underestimate of the concentration of the mass profile. The virial mass estimate, primarily sensitive to distortions at large radius relative to the cluster center, is relatively unbiased by this effect. In fact, the ratio between the Subaru and CFHT profiles for A963 may demonstrate this effect; the ratio is unity around the virial radius and decreases with decreasing radius.

The source redshift controls the overall amplitude of the distortion signal. In general, the claimed error in the average distance ratio is only 5\%-10\%. Different observers use different approaches to estimating the source redshift. Hoekstra (2007) and Okabe et al. (2010) use photometric redshifts to assess the source redshift. Okabe \& Umetsu (2008) assume that the sources are at redshift one in all cases.

Because weak lensing measures the total mass within the lensing kernel and projected within the aperture, foreground and/or background structures along the line of sight affect the measurement (e.g., Hoekstra 2001, 2003; White et al. 2002; de Putter \& White 2005; Hoekstra et al. 2011). Umetsu et al. (2011) show (their Figure 1) that the cosmic noise is an increasing fraction of the lensing signal at larger distance from the cluster center. Oguri et al. (2010) and Okabe et al. (2010) argue that any biases introduced by projected large-scale structure are generally insignificant. In their study of A2261, Coe et al. (2012) remove superposed background structures; this procedure reduces the virial mass by only $\sim 7 \%$ and increases the concentration by only $\sim 5 \%$. However, the effect can be large in some cases; we demonstrate a textbook case in Section 5.

Another issue is departure from spherical symmetry. Most masses derived from weak lensing data are based on azimuthally averaged (one-dimensional (1D)) profiles. Oguri et al. (2010) and Okabe et al. (2010) make full use of the two-dimensional (2D) weak lensing data and compare the shear pattern with predictions of elliptical models. Oguri et al. (2010, Figure 7) show that the 1D and 2D virial masses are remarkably consistent with one another: they differ by only $10 \%$ in the mean and the scatter is $\lesssim 20 \%$. Although Oguri et al. (2010) provide 2D weak lensing profiles for some of the HeCS clusters, we use the 1D profiles because they are available for 18/19 clusters and because the caustic technique also assumes spherical symmetry. For the cluster A689 where no 1D profile is available, we use the 2D profile from Oguri et al. (2010).

In fitting the tangential weak lensing profile, Okabe et al. (2010) take the virial mass and halo concentration as free parameters. Okabe \& Umetsu (2008) use the projected mass profile also with the virial mass and concentration as free parameters. In contrast, Hoekstra (2007) measures the projected mass, deprojects the three-dimensional (3D) mass, and then assumes a fixed mass-concentration relation. These differences may explain some of the differences in the profile ratios shown in Figure 2. The ratio between the Subaru and CFHT mass profile observations for A267 and A2219 may decline with radius as result of the larger concentration obtained when the two fitting parameters are free. Lemze et al. (2008) incorporate strong lensing data into their mass profile for A1689 derived from $H S T$ data; they thus have greater resolution on small scales possibly yielding a more concentrated profile than weak lensing data alone (Figure 2).

Less important issues affecting the weak lensing profiles include differences in the identification of the cluster center and errors in measurement of the shapes of the sources. Centering errors are generally small and various methods of measuring the shapes of the sources differ by $\sim 10 \%$.

Regardless of the source of the differences, the difference between lensing profiles for a single cluster is comparable to the difference between the caustic and lensing mass estimates (see Section 4). We discuss the fundamental limits for the caustic technique in Section 3.

\section{CLUSTER MASS PROFILES: CAUSTICS}

For comparison with the weak lensing profiles, we use the caustic technique (DG97; D99) to derive mass profiles from dense kinematic data for each of the clusters.

Like weak lensing, the caustic technique makes no assumption about the dynamical equilibrium of the system. In contrast with weak lensing, the caustic technique measures the $3 \mathrm{D}$ distribution of mass rather than the projected mass. The caustic technique also extends to large radius and provides a test of weak lensing results outside the virial radius. Structure along the line of sight is generally resolved by the redshift survey; the caustic technique is thus insensitive to it. Like the most straightforward applications of weak lensing, the caustic technique assumes spherical symmetry. More sophisticated applications of weak lensing mass estimates do not necessarily assume azimuthal symmetry; they provide an estimate of the projected surface mass density as a function of position on the sky (see Okabe et al. 2010; Oguri et al. 2010).

In redshift space, a cluster of galaxies appears as a trumpetshaped pattern (Kaiser 1987; Regös \& Geller 19889). DG97 and D99 demonstrated that for clusters forming hierarchically, the boundaries of this sharply defined pattern (termed caustics) in redshift space (a projection of phase space) can be identified with the escape velocity from the cluster. This identification provides a route to estimation of the cluster mass profile assuming spherical symmetry.

The amplitude of the caustics $A(r)$ is half the distance between the boundaries of the cluster in redshift space. With the assumption of spherical symmetry the gravitational potential $\phi(r)$ and the caustic amplitude $A(r)$ are related by

$$
A^{2}(r)=-2 \phi(r) \frac{1-\beta(r)}{3-2 \beta(r)},
$$

where $\beta(r)$ is the anisotropy parameter, $\beta(r)=1-\sigma_{\theta}^{2}(r) / \sigma_{r}^{2}(r)$ where $\sigma_{\theta}$ and $\sigma_{r}$ are, respectively, the tangential and radial velocity dispersions.

DG97 show that the mass of a spherical shell within the infall region is the integral of the square of the caustic amplitude $A(r)$ :

$$
G M(<r)-G M\left(<r_{0}\right)=F_{\beta} \int_{r}^{r_{0}} A^{2}(x) d x,
$$

where $F_{\beta} \simeq 0.5$ is a filling factor with a value estimated from numerical simulations. We approximate $F_{\beta}$ as a constant; variations in $F_{\beta}$ with radius lead to some systematic uncertainty in the mass profile we derive from the caustic technique. We include these issues in our assessment of the intrinsic uncertainties and biases in the technique (Serra et al. 2011).

The first step in applying the caustic technique is identification of the cluster center. We isolate the cluster initially by selecting all of the galaxies in our redshift survey that lie within $10 \mathrm{~h}^{-1} \mathrm{Mpc}$ and $5000 \mathrm{~km} \mathrm{~s}^{-1}$ of the nominal X-ray cluster center. We then construct a binary tree based on pairwise estimated binding energies. We use the tree to identify the largest cluster in 

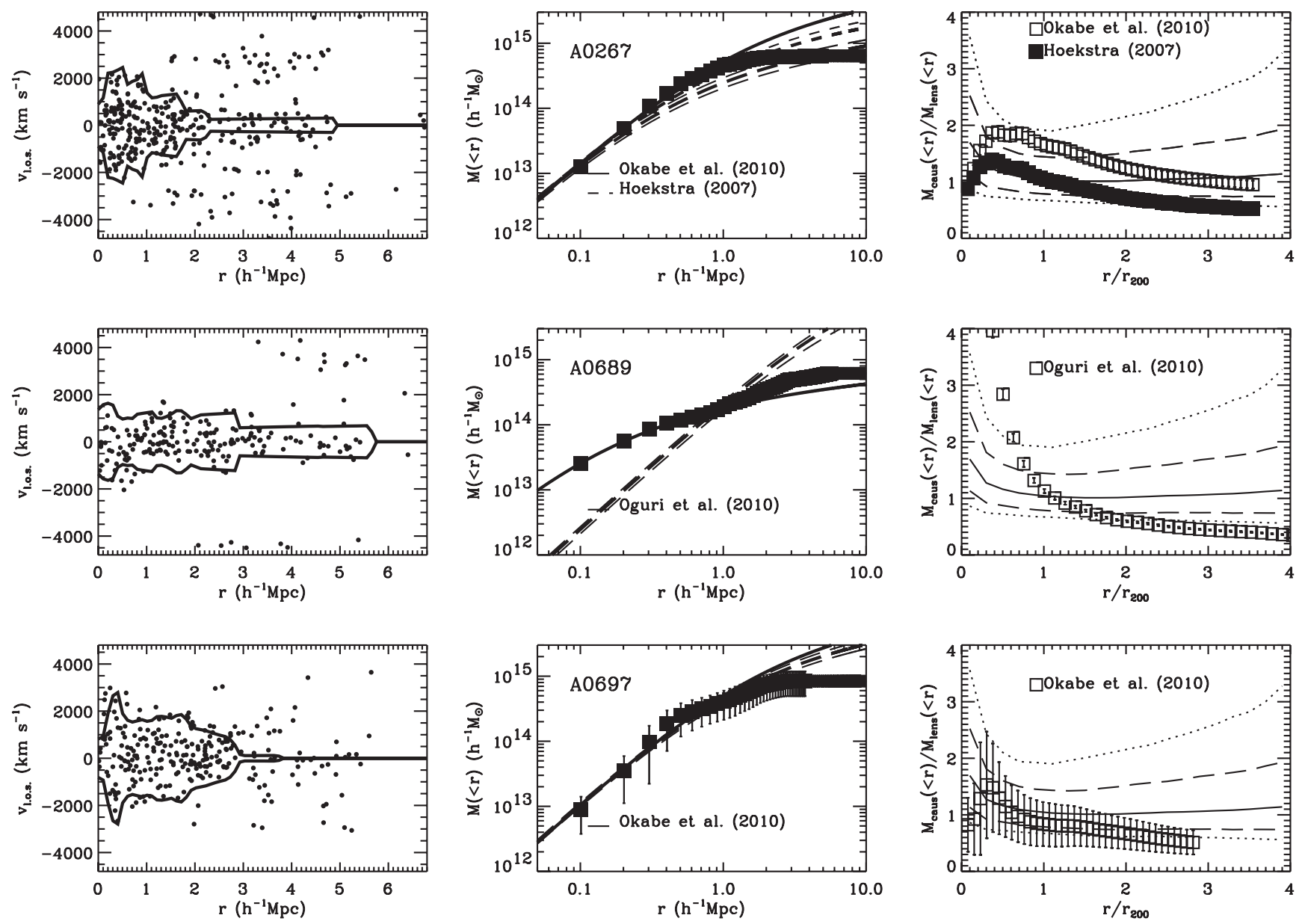

Figure 3. Left column: caustic diagrams for individual clusters in the HeCS sample. The vertical axis is the rest frame line-of-sight velocity relative to the hierarchical cluster center; the horizontal axis is the projected distance from the hierarchical center. Each point represents a galaxy with a redshift in the HeCS sample, the curves are the caustics. Center column: mass profiles for individual clusters. Points with error bars are the caustic estimate, the solid curve is an NFW fit to the caustic profile within $1 h^{-1} \mathrm{Mpc}$, and dashed curves are the NFW fits (heavy) and $1 \sigma$ errors (light) for the corresponding weak lensing profiles with the source indicated in the panel. Right column: ratio of caustic mass profiles and weak lensing mass profiles for individual clusters. Points with error bars give the ratio. For cases with more than one weak lensing profile, the symbols give the source as indicated in the panel. The solid curve is the ratio between the caustic profile and true mass profiles computed from $N$-body simulations (Serra et al. 2011). The dashed and dotted curves are the $1 \sigma$ and $2 \sigma$ limits on the ratio derived in the $N$-body simulations.

the field and we adaptively smooth the distribution of galaxies within this cluster to identify its center (see D99 and Serra et al. 2011 for detailed descriptions of this process).

Once we have identified a center we can plot the distribution of galaxies in azimuthally summed phase space. This effective azimuthal averaging smooths over small-scale substructure particularly at large projected radius. Figures 3-9 show the distribution of galaxies in the rest frame line-of-sight velocity versus projected spatial separation plane for the 19 clusters in this study. The expected trumpet-shaped pattern centered on the mean cluster velocity is evident in all cases.

To measure the amplitude $A(r)$ of the phase-space signature of the cluster, we smooth the patterns in Figures 3-9 and identify a threshold in phase-space density as the edge of the caustic envelope. We define the threshold $\kappa$ by solving the equation $\left|\left\langle v_{\text {esc }}{ }^{2}\right\rangle_{\kappa, R}-\left\langle v^{2}\right\rangle_{R}\right|=0$ where $R$ is a virial-like radius and $v_{\text {esc }}$ is the escape velocity at radius $R$ (D99 and Serra et al. 2011).

In a real cluster, the values of the upper, $A^{+}(r)$, and lower, $A^{-}(r)$, caustic amplitude in the redshift diagram are not identical. Because the caustics of a spherical system are identical, we adopt the smaller value of the two values $A^{+}(r)$ and $A^{-}(r)$ as our estimate of $A(r)$.

To compute the shape of the caustics, according to the algorithm described in D99, we choose a smoothing parameter $q=25$ where $q$ is the scaling between the velocity and radial smoothing in the adaptive kernel estimate of the underlying phase-space distribution. For example, a particle with a smoothing window of $0.04 \mathrm{~h}^{-1} \mathrm{Mpc}$ in the spatial direction has a $100 \mathrm{~km} \mathrm{~s}^{-1}$ smoothing window along the velocity direction. Variations of a factor of two in $q$ have essentially no effect on the results (Geller at al. 1999; Rines et al. 2000, 2002; Rines \& Diaferio 2006). The solid lines in Figures 3-9 show the caustics we compute with this procedure.

D99 and Serra et al. (2011) investigate the caustic method in detail; they evaluate the uncertainties and systematic biases by applying the technique to clusters in $\mathrm{N}$-body simulations. On average the caustic recovers the mass profile without any systematic bias and a $1 \sigma$ error of about $\sim 50 \%$ in the range $\sim(0.6-4) r_{200}$ ( $r_{200}$ is the radius that encloses a mean density 200 times the critical density). There is a bias toward overestimating the mass at radius smaller than $\sim 0.6 r_{200}$ by $\sim 70 \%$ at most; this bias results primarily from the assumption of a constant $\mathrm{F}_{\beta}$. Projection effects, a limitation on every mass estimation technique at some level, are the main source of scatter in the caustic mass estimates.

Table 2 summarizes the results of the application of the caustic technique to the $19 \mathrm{CIRS} / \mathrm{HeCS}$ clusters. The caustic mass profiles allow direct estimation of $r_{200}^{\text {caus }}$ and the mass within 

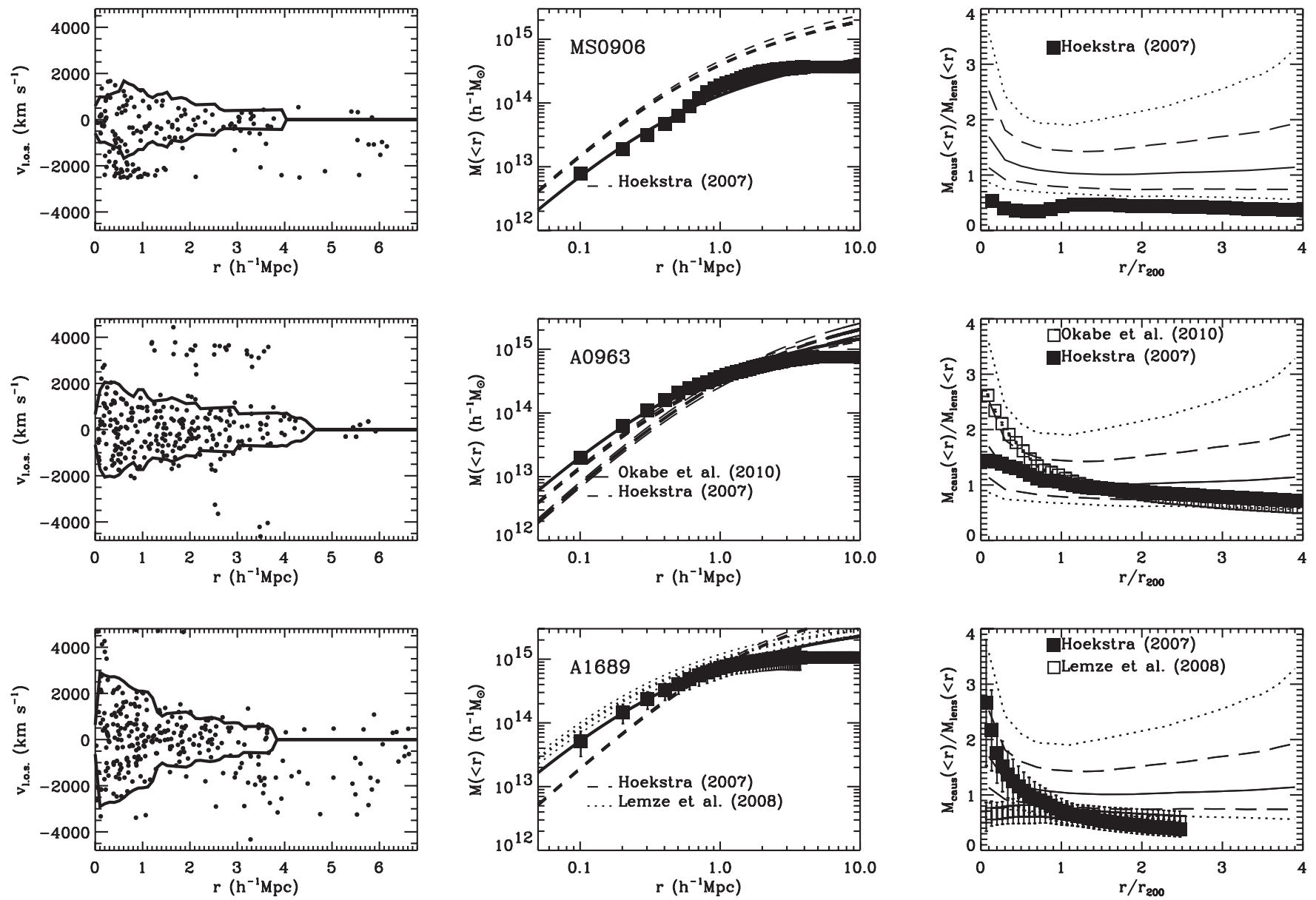

Figure 4. Caustic diagrams (right column), mass profiles (center column), and caustic to lensing profile ratios (left column) for individual clusters in the HeCS sample as in Figure 3.

Table 2

HeCS and CIRS Caustic Mass Estimates ${ }^{\mathrm{a}}$

\begin{tabular}{|c|c|c|c|c|c|c|c|}
\hline Cluster & $z$ & $M_{200}^{\text {caus }} / 10^{14} h^{-1} M_{\odot}$ & $M_{200}^{\mathrm{NFW}} / 10^{14} h^{-1} M_{\odot}$ & $r_{200}^{\text {caus }} / h^{-1} \mathrm{Mpc}$ & $r_{200}^{\mathrm{NFW}} / h^{-1} \mathrm{Mpc}$ & $c_{200}=r_{200} / r_{\mathrm{rs}}$ & $N_{\text {caus }}$ \\
\hline A0267 & 0.229 & $4.92 \pm 0.26$ & $7.5 \pm 1.3$ & $1.19 \pm 0.02$ & $1.37 \pm 0.08$ & $1.80 \pm 0.16$ & 226 \\
\hline A0689 & 0.279 & $1.54 \pm 0.05$ & $1.59 \pm 0.07$ & $0.80 \pm 0.01$ & $0.80 \pm 0.01$ & $9.05 \pm 0.35$ & 163 \\
\hline A0697 & 0.281 & $4.4 \pm 2.0$ & $7 \pm 14$ & $1.13 \pm 0.24$ & $1.33 \pm 0.90$ & $1.1 \pm 1.2$ & 185 \\
\hline MS0906 & 0.177 & $1.47 \pm 0.25$ & $0.95 \pm 0.19$ & $0.81 \pm 0.07$ & $0.70 \pm 0.05$ & $2.31 \pm 0.26$ & 101 \\
\hline A0963 ${ }^{b}$ & 0.204 & $4.00 \pm 0.04$ & $4.35 \pm 0.08$ & $1.12 \pm 0.01$ & $1.15 \pm 0.01$ & $3.69 \pm 0.04$ & 211 \\
\hline A1689 & 0.184 & $8.6 \pm 1.9$ & $9.6 \pm 5.8$ & $1.46 \pm 0.12$ & $1.51 \pm 0.30$ & $7.1 \pm 3.5$ & 210 \\
\hline $\mathrm{A} 1750^{\mathrm{c}}$ & 0.085 & $3.00 \pm 0.09$ & $2.68 \pm 0.31$ & $1.06 \pm 0.01$ & $1.02 \pm 0.04$ & $2.69 \pm 0.20$ & 398 \\
\hline A1758 & 0.276 & $2.22 \pm 0.77$ & $2.0 \pm 1.2$ & $0.90 \pm 0.13$ & $0.87 \pm 0.17$ & $4.9 \pm 2.3$ & 143 \\
\hline A1763 & 0.231 & $12.4 \pm 1.4$ & $12.6 \pm 3.8$ & $1.62 \pm 0.08$ & $1.63 \pm 0.16$ & $5.7 \pm 1.3$ & 237 \\
\hline A 1835 & 0.251 & $8.41 \pm 0.53$ & $11.6 \pm 1.3$ & $1.41 \pm 0.03$ & $1.57 \pm 0.06$ & $3.36 \pm 0.23$ & 219 \\
\hline A1914 & 0.166 & $4.75 \pm 0.13$ & $4.62 \pm 0.23$ & $1.20 \pm 0.01$ & $1.19 \pm 0.02$ & $11.76 \pm 0.63$ & 255 \\
\hline $\mathrm{A} 2009^{\mathrm{b}}$ & 0.152 & $3.49 \pm 0.16$ & $3.16 \pm 0.29$ & $1.09 \pm 0.02$ & $1.05 \pm 0.03$ & $5.82 \pm 0.44$ & 195 \\
\hline A2034 & 0.113 & $5.00 \pm 0.03$ & $5.60 \pm 0.08$ & $1.25 \pm 0.01$ & $1.29 \pm 0.01$ & $3.00 \pm 0.03$ & 182 \\
\hline $\mathrm{A} 2142^{\mathrm{c}}$ & 0.090 & $2.9 \pm 1.0$ & $2.7 \pm 3.2$ & $1.04 \pm 0.18$ & $1.02 \pm 0.41$ & $2.5 \pm 1.9$ & 248 \\
\hline A2219 & 0.226 & $8.9 \pm 1.9$ & $9.4 \pm 5.2$ & $1.46 \pm 0.14$ & $1.48 \pm 0.27$ & $6.2 \pm 2.8$ & 461 \\
\hline RXJ1720 & 0.160 & $4.44 \pm 0.24$ & $4.28 \pm 0.43$ & $1.18 \pm 0.03$ & $1.16 \pm 0.04$ & $9.6 \pm 0.9$ & 376 \\
\hline A2261 & 0.224 & $2.60 \pm 0.73$ & $2.3 \pm 2.4$ & $0.97 \pm 0.12$ & $0.93 \pm 0.32$ & $2.2 \pm 1.5$ & 228 \\
\hline A2631 & 0.277 & $3.77 \pm 0.66$ & $3.8 \pm 1.8$ & $1.07 \pm 0.08$ & $1.07 \pm 0.17$ & $2.62 \pm 0.79$ & 173 \\
\hline RXJ2129 & 0.234 & $5.6 \pm 1.0$ & $4.5 \pm 1.7$ & $1.24 \pm 0.11$ & $1.16 \pm 0.15$ & $9.6 \pm 3.5$ & 325 \\
\hline
\end{tabular}

Notes.

${ }^{\text {a }} M_{200}^{\text {caus }}$ and $r_{200}^{\text {caus }}$ from the caustic mass profile and the best-fit NFW parameters to the caustic mass profiles within $1 h^{-1}$ Mpc. The last column lists the number of galaxies within the caustics in the redshift diagram.

${ }^{\mathrm{b}}$ Indicates that the source catalog for the weak lensing measurement might include significant contamination by cluster members (Okabe et al. 2010, Table 3).

${ }^{\mathrm{c}}$ Denotes a cluster in the CIRS sample. All other systems are in the HeCS sample. 

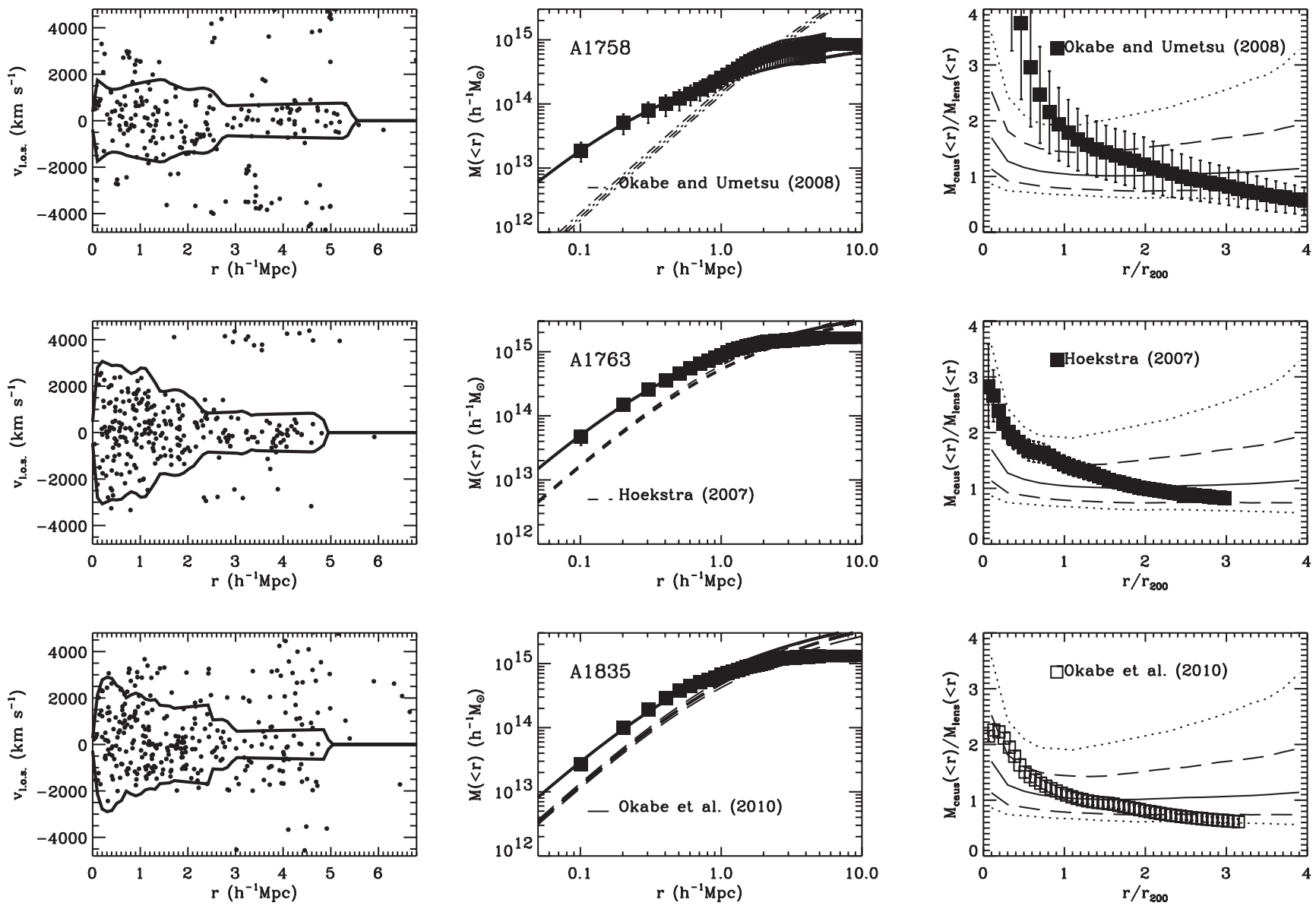

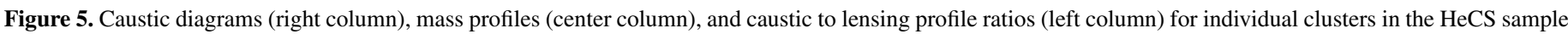
as in Figure 3.

this radius, $M_{200}^{\text {caus }}$. Table 2 lists $r_{200}^{\text {caus }}$ and $M_{200}^{\text {caus }}$. The table also lists $N_{\text {caus }}$, the number of galaxies within the caustics. These clusters are all sampled well enough for robust application of the technique (see Serra et al. 2011).

We also fit the caustic mass profiles to the analytic NFW profile within $1 h^{-1} \mathrm{Mpc}$ from the cluster center, a typical radius delimiting the region where the NFW profile is expected to hold:

$$
M(<r)=\frac{M(a)}{\ln (2)-1 / 2}\left[\ln \left(1+\frac{r}{a}\right)-\frac{r}{a+r}\right],
$$

where $a$ is the scale radius and $M(a)$ is the mass with the scale radius. We fit $M(a)$ rather than the characteristic density $\delta_{c}\left(M(a)=4 \pi \delta_{c} \rho_{c} a^{3}[\ln (2)-1 / 2]\right.$ where $\rho_{c}$ is the critical density) because $M(a)$ and $a$ are much less correlated than $\delta_{c}$ and $a$ (Mahdavi et al. 1999). Table 2 lists $M_{200}^{\mathrm{NFW}}, r_{200}^{\mathrm{NFW}}$, and the NFW concentration parameter $c_{200}$.

\section{MASS PROFILES: CAUSTICS AND WEAK LENSING}

Weak lensing and the caustic technique are fundamentally different measures of the mass distribution in a cluster. Weak lensing measures the total projected mass density within the lensing kernel; the caustic technique measures the mass within a given radius modulo the effects of both geometric and velocity anisotropy. Both techniques enable the measurement of a mass profile to large projected radius and both are independent of the assumption of dynamical equilibrium.

Comparison of observed mass profiles derived from these two techniques may elucidate the uncertainties and systematic problems in both approaches. Independent structures along the line of sight and within the lensing kernel bias the weak lensing mass profiles (e.g., Hoekstra 2003; Coe et al. 2012). Application of the caustic mass estimation technique is limited by departures from spherical symmetry (extension along the line of sight is also a problem for weak lensing mass estimates) and by lack of knowledge of the velocity anisotropy. The assumption of a constant $F_{\beta}$ (which includes the velocity anisotropy information) leads to an overestimate of the mass at small radii within $\sim 0.6 r_{200}$ (D99; Serra et al. 2011).

Here we compare caustic and weak lensing profiles with an eye toward exploring the relative systematic issues in the lensing and kinematic mass estimates. Figures 3-8 show redshift space diagrams (left column) for the $17 \mathrm{HeCS}$ clusters ordered in right ascension. Figure 9 shows the redshift space diagrams for the two CIRS clusters. The solid lines in these diagrams locate the caustics according to the prescription of D99. The central panel shows the caustic mass profile (points with error bars), the NFW fit to the caustic profile (solid line) and the NFW fits quoted for the lensing profiles from the literature (dashed and dash-dotted lines). The radial extent of the weak lensing profiles is limited to radii $\lesssim 1.5 r_{200}$ by the areal coverage of the imaging data. Thus for radii $\gtrsim 1.5 r_{200}$ the weak lensing profiles in Figures 3-9 are extrapolations of the NFW fit to the weak lensing profile; the direct caustic mass profile measurements extend to these large radii as indicated by the solid squares.

In most cases the caustic mass profile lies above the lensing mass profile at small radius and below at large radius. The profiles generally cross around the virial radius where the 

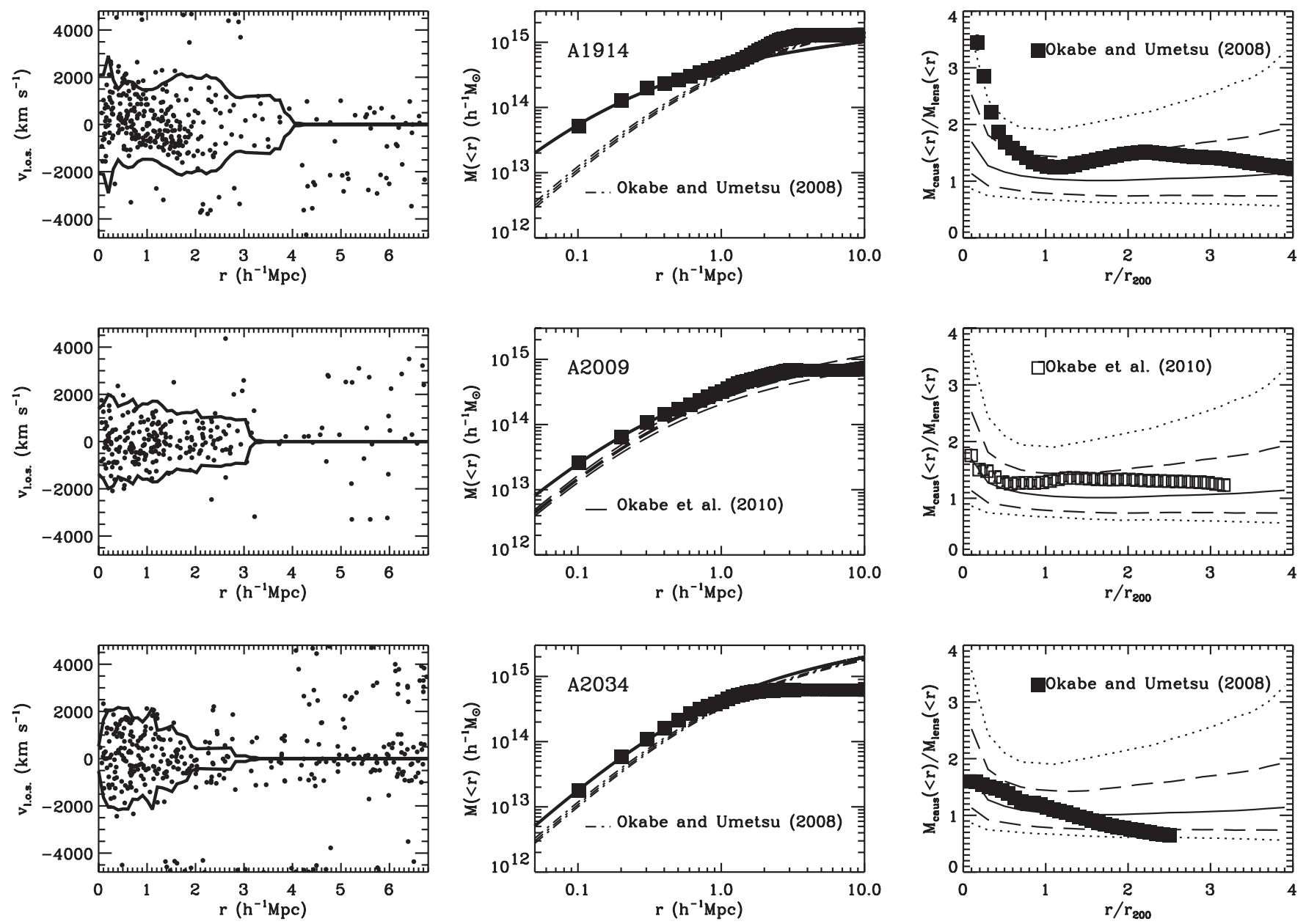

Figure 6. Caustic diagrams (right column), mass profiles (center column), and caustic to lensing profile ratios (left column) for individual clusters in the HeCS sample as in Figure 3.

two techniques should yield the same result. In some cases (particularly A2631) the caustic mass profile tracks the weak lensing profile. In two cases, the caustic mass profile lies below the lensing mass profile throughout the range (MS0906 +11 and A2142). There is no obvious reason for the discrepancy in A2142; we discuss the remarkable case of MS0906 +11 in Section 5 .

The data points with error bars in the right panel for each cluster show the ratio between the caustic mass profile, $M_{\text {caus }}(<r)$, and the weak lensing profile, $M_{\text {lens }}(<r)$ as a function of projected radius, $r / r_{200}$. For the caustic mass profile, we use $r_{200}$ derived from the NFW fit. The solid, dashed, and dotted curves are the median ratio between the caustic profile and the true profile of a sample of synthetic clusters extracted from an $N$-body simulation (solid curve), along with the $1 \sigma$ (dashed) and $2 \sigma$ (dotted) error range (Serra et al. 2011). These curves demonstrate the results described above in Section 3. This comparison shows that the caustic profile overestimate in the central region is a systematic issue in the technique. However, the underestimate at large radius relative to weak lensing does not reflect an inherent bias in the caustic technique. This latter issue may indicate that the profiles at large radius are genuinely steeper than the extrapolated NFW weak lensing profiles (see Rines et al. 2012).

For the A267, A1689, and A963 we show the ratio between the caustic mass and the weak lensing mass for two different observations (Figure 2). These plots underscore the conclusion from Figure 2. At small radius, the differences between weak lensing profiles derived by different observers on different facilities can be comparable with the difference between the caustic mass profile and the weak lensing profile.

\subsection{Comments on Individual Clusters}

All of the clusters we consider are selected from X-ray cluster catalogs. HeCS and CIRS are X-ray flux-limited samples of clusters. We comment here on the known "irregularities" of nine of the clusters in our weak lensing comparison sample. We reserve discussion of a tenth system, MS0906+11, for Section 5 .

A689. This cluster is actually below the flux limit of the HeCS sample. The original inclusion of A689 in the ROSAT Brightest Cluster Sample (Ebeling et al. 1998) resulted from a superposed BL Lac object later pinpointed by a Chandra observation (Giles et al. 2012). The low mass of this cluster is consistent with the lower X-ray luminosity (Rines et al. 2012). This issue should not have any effect on the comparison of caustic and weak lensing mass profiles.

Okabe et al. (2010) do not fit a mass profile to their weak lensing data for this cluster because the mass map shows prominent substructures. Oguri et al. (2010) provide the fit we use for comparison with the caustic mass profile, but caution that the NFW model fit is unacceptable. Their value of $c_{\mathrm{vir}}=0.41$ does not make physical sense (as they indicate).

These issues in interpreting the weak lensing data may account for the very large difference between the caustic and 

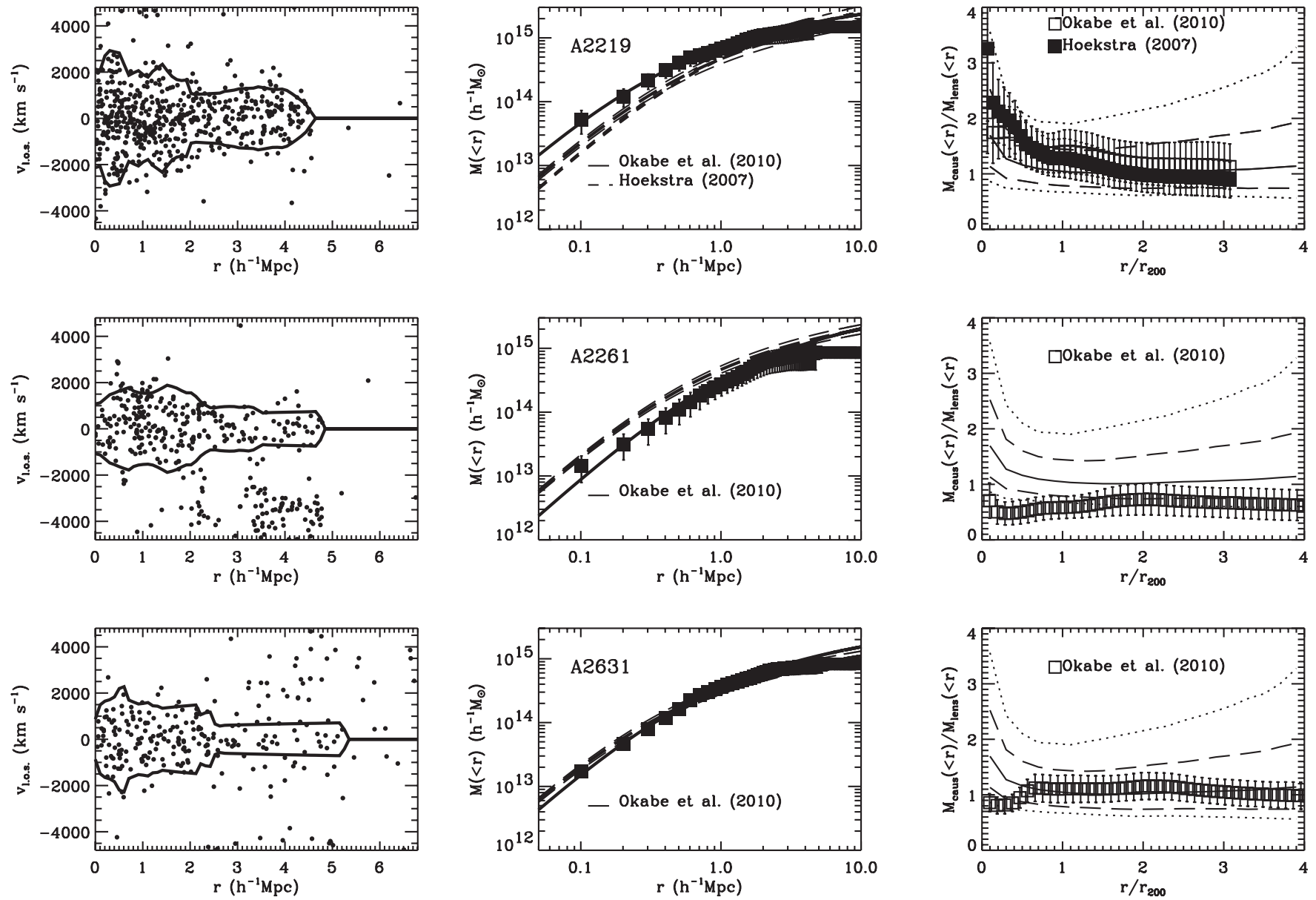

Figure 7. Caustic diagrams (right column), mass profiles (center column), and caustic to lensing profile ratios (left column) for individual clusters in the HeCS sample as in Figure 3.
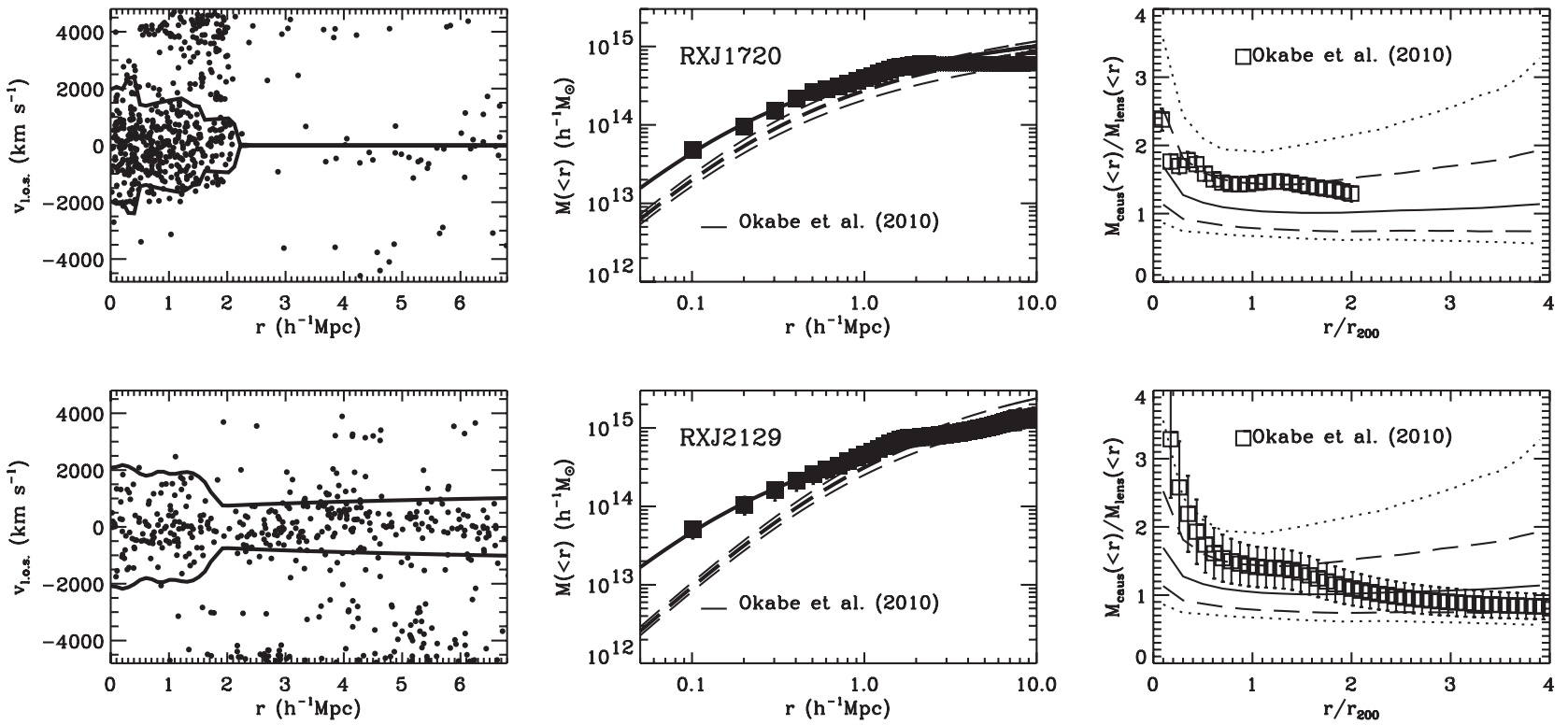

Figure 8. Caustic diagrams (right column), mass profiles (center column), and caustic to lensing profile ratios (left column) for individual clusters in the HeCS sample as in Figure 3.

weak lensing profiles within $\sim r_{200}$ (Figure 3 ); the caustics, in contrast, are stable and well defined. The caustic mass profile is insensitive to the substructure. The ratio between the caustic and weak lensing profiles is among the few that are well outside the ratio between the true and caustic mass profiles derived from $N$-body calibrations.

A963. Unlensed foreground galaxies probably dilute the lensing signal for this cluster (Okabe et al. 2010). Thus the 

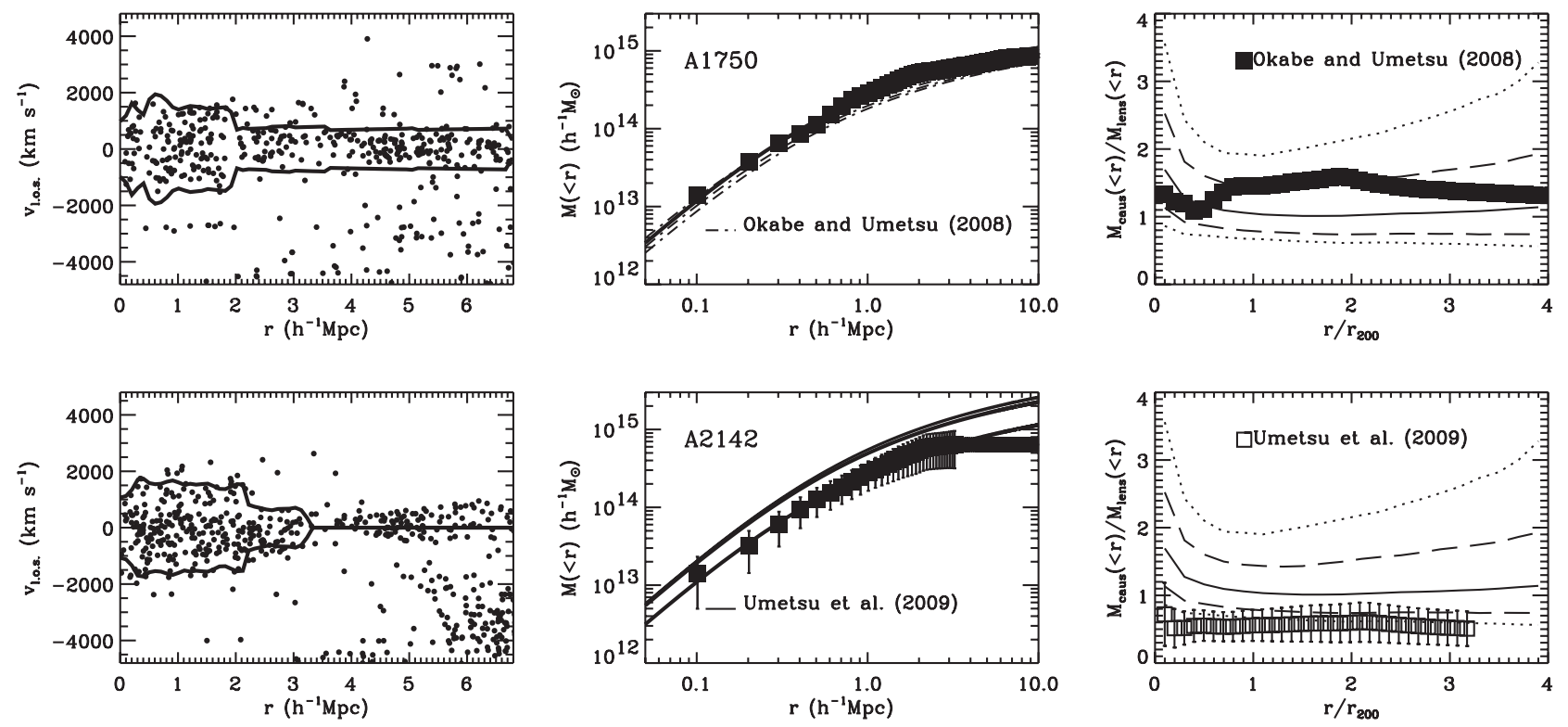

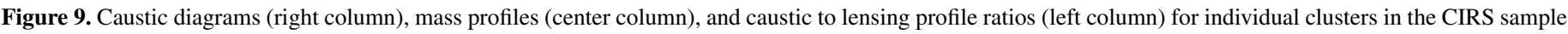
as in Figure 3.

lensing mass profile may be underestimated within the virial radius.

A1750. A1750 is binary X-ray cluster (e.g., Forman et al. 1981; Belsole et al. 2004). We compare our caustic mass estimate with Okabe \& Umetsu's (2008) lensing profile for the most luminous X-ray component, A1750C. The hierarchical center for the caustic mass calculation is very close to this X-ray peak in both position and velocity.

A1758. A1758 is a merger of multiple X-ray clusters. Böhringer et al. (2000) and others separate the cluster into A1758N and A1758S. The lensing analysis of Okabe \& Umetsu (2008) shows that A1758N consists of two separate clusters A1758N:C and A1758N:SE. They center their mass profile on A1758N:C (labeling it as A1758N). Because A1758 is one of the most distant clusters in $\mathrm{HeCS}$, it is not very densely sampled. Nonetheless, the caustics are visible and the hierarchical center is nearly coincident with the brightest galaxy in A1758N:C. This center is consistent with the center for the weak lensing analysis.

The weak lensing map of Okabe \& Umetsu shows prominent substructures. As in the case of A689, these substructures may be responsible, at least in part, for the very large difference between the caustic and weak lensing mass profiles within $r_{200}$.

A1914. X-ray observations suggest that A1914 is a major merger in progress (Govoni et al. 2004). Although the caustic pattern is visible in Figure 6, the distribution of galaxies within the region is odd. In spite of this odd distribution, the caustic and weak lensing agree to within the expected $1 \sigma$ scatter for the caustic mass profiles around $r_{200}$.

A2009. As in the case of A963, unlensed foreground galaxies probably dilute the lensing signal for this cluster (Okabe et al. 2010). Thus the lensing mass profile may be underestimated within the virial radius.

A2034. A2034 (Kempner et al. 2003) is a cold front cluster. The mass map (Okabe \& Umetsu 2008) shows complex structure perhaps indicating a merger of components responsible for the cold front. The caustics are cleanly determined.

A2142. A2142 is the prototype cold front cluster initially discovered by Markevitch et al. (2000). The weak lensing map by Umetsu et al. (2009) shows complex structure as for A2034.
A2261. A2261 contains a very unusual brightest cluster galaxy (BCG) with a large central velocity dispersion and an extended flat core (Postman et al. 2012). The sampling of this cluster with the 300 fiber Hectospec instrument is restricted by bright stars in the field. Oddly, the hierarchical center of the cluster identified by the D99 procedure is $\sim 6^{\prime}$ south of the BCG and the BCG is offset from the cluster mean by about $\sim 400 \mathrm{~km}$ $\mathrm{s}^{-1}$ in the rest frame, as we reported in Coe et al. (2012). Serra et al. (2011) have a slightly different procedure for cutting the binary tree; this algorithm yields the cluster center on the BCG. A2261 is a complex system and the D99 center is coincident with a cluster substructure. These two centers are relatively close to each other and the caustic mass profile is insensitive to the final center choice. Here we show the profile centered on the BCG.

The total sample of 19 clusters appears to include systems in a broad range of dynamical states. When examined in enough detail, every cluster of galaxies reveals some kind of complexity. Substructure enters the caustic and lensing estimates through its effect on the determination of the position of the caustics and its effect on the fitting of the NFW weak lensing profile. We note that the two clusters A689 and A1758 with the largest ratios of caustic to weak lensing mass profiles at small radii are dominated by substructure and/or sampling issues. In spite of these issues, we take all of the caustic and lensing profiles at face value to assess the relative measurements of cluster mass profiles in Section 6.

\section{MS0906: A REMARKABLE EXAMPLE OF SUPERPOSITION ALONG THE LINE OF SIGHT}

Structures projected along the line of sight are a fundamental limitation on the accuracy of weak lensing profiles (e.g., Hoekstra 2001, 2003; White et al. 2002; de Putter \& White 2005; Hoekstra et al. 2011). Here we show a remarkable case where two massive clusters at different redshifts share nearly the same central position on the sky. We demonstrate that this remarkable superposition leads to very substantial contamination of the weak lensing mass profile published by Hoekstra (2007). In fact, the weak lensing mass is approximately the sum 


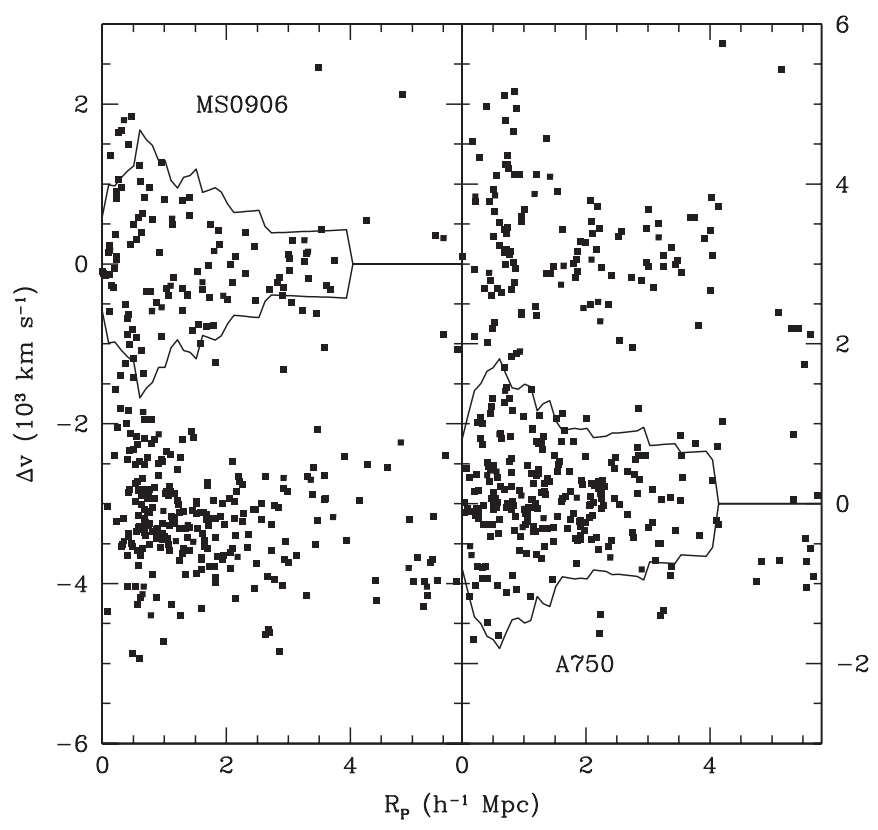

Figure 10. Caustic diagrams for MS0906 and A750 superposition. On the left, MS0906 sets the zero point for the relative line-of-sight velocities; on the right A750 sets the zero point.

of the masses of the two clusters nearly aligned along the line of sight.

MS0906+11 is an extended X-ray source discovered in the Einstein Slew Survey (Elvis et al. 1992). A750, a less luminous extended X-ray source, is centered only $5^{\prime}\left(0.63 h^{-1} \mathrm{Mpc}\right)$ from the X-ray center of MS0906+11 (see Figure 3.39 of Maughan et al. 2008). The mean redshifts of the two clusters are similar: $z=0.1767$ for MS0906+11 and $z=0.1640$ for A750. On the basis of a sparse survey, Carlberg et al. (1996, p. 37) wrote that MS0906 "appears to be an indistinct binary in redshift space."

Dense HeCS spectroscopy demonstrated cleanly that there are two distinct clusters along the line of sight (Rines et al. 2012). Figure 10 shows the caustic diagrams for the two clusters; the two patterns are readily visible. In the left-hand panel MS0906+11 determines the zero point. In the right-hand panel, A750 determines the zero point. In the rest frame the two clusters are separated by $3250 \mathrm{~km} \mathrm{~s}^{-1}$.

There is some confusion in the literature about the identification of A750 and MS0906+11 on the sky. Okabe et al. (2010) show maps of the surface mass density and galaxy red sequence luminosity density for A750 (their Figure 30). Actually, in their panel B, the concentration marked C is MS0906+11, not A750. Their NW1 component is A750. The cluster MS0906+11 has a greater central surface mass density (and presumably greater mass) than A750 (NW1); the red sequence luminosity density (galaxies on the red sequence with $R_{A B}<22$ ) appears to be greater for A750. Because of the complexity of the system, Okabe et al. (2010) do not report a weak lensing mass for the apparently complex system they call A750.

For the HeCS observations of the MS0906+11 field, we selected galaxies within $0.3 \mathrm{mag}$ of the red sequence at the mean redshift of MS0906+11 and with SDSS $r=16-21$. Figure 11 shows that the difference between the red sequences of MS0906+11 (blue open squares) and A750 (open red triangles) is subtle. We centered the Hectospec pointings on the center of MS0906+11; thus any position bias (if any) in the spectroscopy favors MS0906+11.

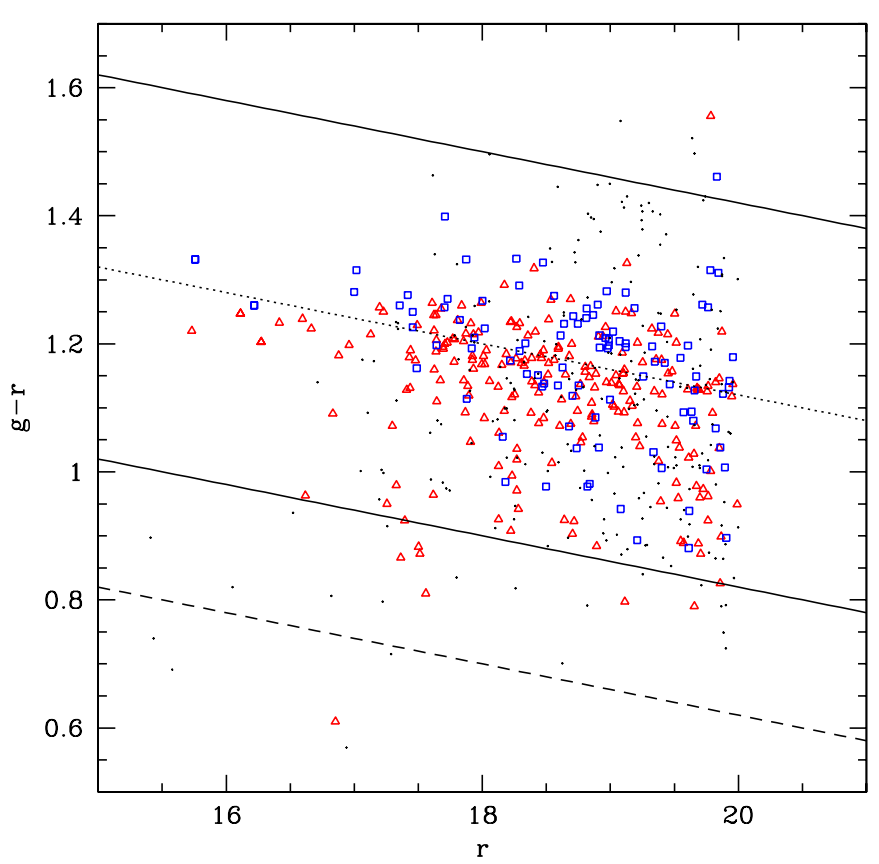

Figure 11. Color-magnitude diagram for MS0906/A750. $r$ magnitudes and $g-r$ color are from the SDSS. Solid lines indicate the main Hectospec target selection; the dashed lines indicate the limits for secondary targets. Red triangles indicate A750 members, blue squares are MS0906+11 members, and black dots are non-members.

(A color version of this figure is available in the online journal.)

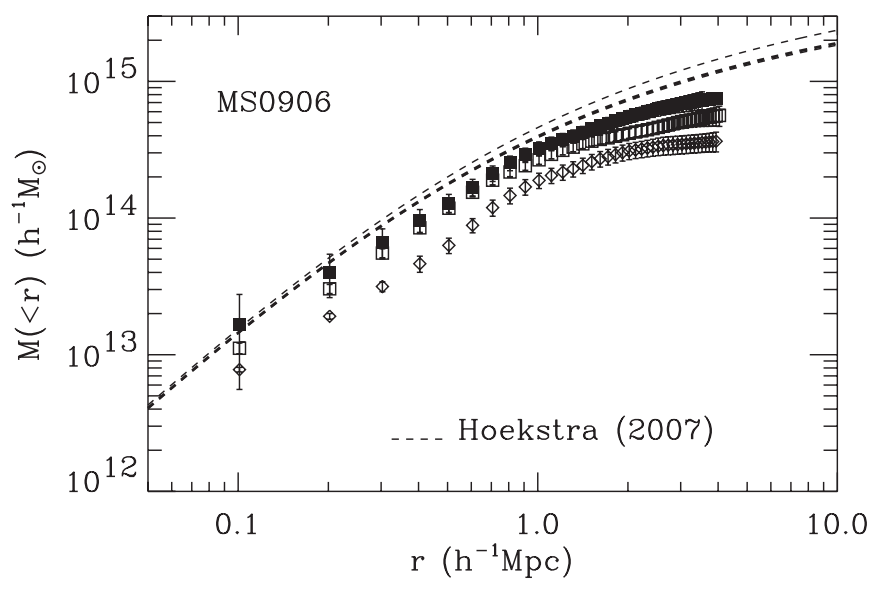

Figure 12. Comparison of the weak lensing mass profile for MS0906 with $1 \sigma$ errors (heavy and light dashed curves, respectively; Hoekstra et al. 2007) with the caustic mass profiles for MS0906 (open diamonds), A750 (open squares), and the effective sum caustic mass profiles for the two superposed clusters taking the $0.6 h^{-1} \mathrm{Mpc}$ offset between the centers into account (solid squares). For $\mathrm{A} 750, z=0.164, M_{200}^{\text {caus }} / 10^{14} h^{-1} M_{\odot}=2.61 \pm 0.17, M_{200}^{\mathrm{NFW}} / 10^{14} h^{-1} M_{\odot}=$ $2.64 \pm 0.57, r_{200}^{\text {caus }} / h^{-1} \mathrm{Mpc}=0.99 \pm 0.03, r_{200}^{\mathrm{NFW}} / h^{-1} \mathrm{Mpc}=0.99 \pm 0.07$, $c_{200}=r_{200} / r_{\mathrm{rs}}=1.92 \pm 0.24$, and $N_{\text {caus }}=225$.

The HeCS data confirm the suggestion of Okabe et al. (2010); the number and total $R$-band luminosity of galaxies in A750 exceeds those in MS0906+11. Within $R_{200}$ and with $M_{r}<-19$ there are 86 galaxies in A750 and only 41 in MS0906+11. The luminosity ratio is $L_{750} / L_{M S 0906} \sim 1.55 \pm 0.29$, consistent with the mass ratio $M_{200, A 750} / M_{200, M S 0906}=1.78 \pm 0.27$.

Figure 12 summarizes the published weak lensing mass determination for MS0906+11 and the caustic masses derived from the HeCS data. For comparison with the lensing mass we sum the caustic masses of A750 and MS0906+11, by taking into account the $0.6 h^{-1} \mathrm{Mpc}$ projected separation between the two 


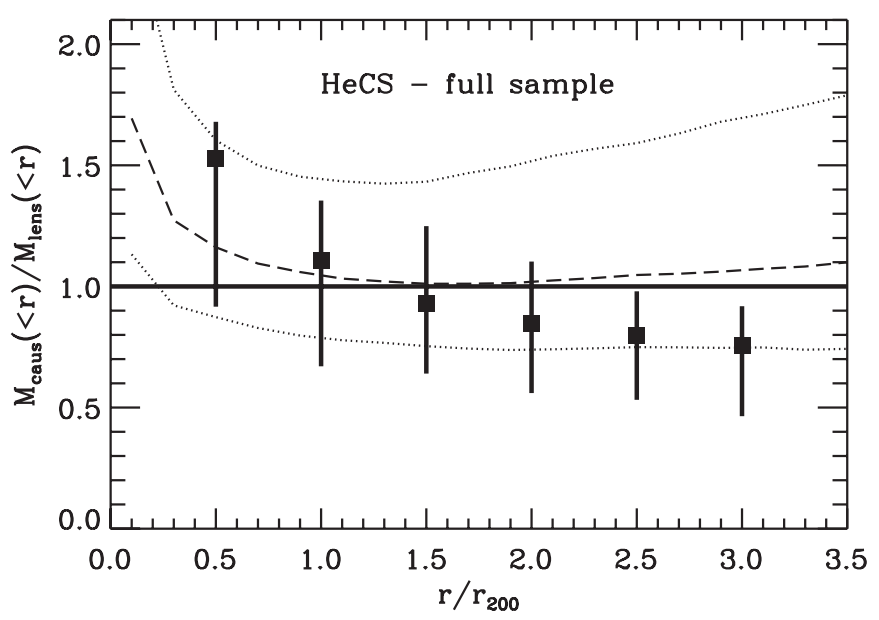

Figure 13. Median (squares) ratio of caustic mass to weak lensing mass as a function of $r / r_{200}$. The bars indicate the interquartile range. The dashed line shows the ratio between the caustic and true mass profiles derived from $N$-body simulations and the dotted lines show the $68 \%$ confidence interval (we also plot these curves in Figures 3-9).

cluster centers. The lensing mass is remarkably close to this sum of the dynamical masses computed from the caustic technique.

\section{DISCUSSION}

Comparison of mass profiles derived from weak lensing and from the caustic technique highlights the relative strengths of the two techniques. Weak lensing measurements have small statistical errors at small radius. The major systematics at small radii (see Section 2.2) can be well controlled. For the caustic technique, the most severe bias occurs in the core where the assumption of a constant form factor (see Section 3 ) is poorest. The caustic technique is insensitive to unrelated superposed structures throughout the radial range of the profile.

At $r_{200}$, the typical error in a weak lensing mass profile is $\sim 20 \%$ for a cluster at $z=0.3$; the error increases to nearly $\sim 30 \%$ at $z=0.1$ (Hoekstra 2003, his Figure 7). Becker \& Kravtsov (2011) consider the systematic error introduced by fitting NFW profiles to weak lensing; depending upon the details of the weak lensing analysis, the NFW fit underestimates the true mass by $\sim 5 \%-10 \%$. For the caustic mass profile the error at $r_{200}$ is $\lesssim 50 \%$. For both techniques, the systematic error in this range appears to be relatively small.

Figure 13 shows the median behavior (and interquartile range) of the relative caustic and lensing mass profiles for our total sample of 19 clusters. For comparison, the dashed line shows the median ratio between the caustic and true mass profiles of clusters extracted from an $\mathrm{N}$-body simulation and the dotted lines show the $68 \%$ confidence interval (we also plot these curves in Figures 3-9). Throughout the radial range we sample, the median ratio of the caustic and weak lensing profiles lies within the $68 \%$ confidence intervals we derive from the $N$-body simulation.

Figure 13 shows that the weak lensing and caustic mass profiles agree stunningly near the virial radius $\left(r_{\mathrm{vir}} \sim 1.3 r_{200}\right)$. At radii less than $r_{\text {vir }}$ the caustic mass profile exceeds the weak lensing profile. If the weak lensing profile is close to the true mass profile, the ratio between the caustic and weak lensing profiles behaves as we would expect based on the $N$-body simulations. In other words, if we chose an average form factor, $\mathrm{F}_{\beta}$, that is a function of radius (not a constant) based on the simulations, the weak lensing and caustic profiles would match to within $\sim 30 \%$ on scales less than $r_{\text {vir }}$.

At radii greater than $r_{\mathrm{vir}}$, the lensing profiles overestimate the mass profile relative to the caustic estimate. At $3 r_{200}$, the comparison suggests that the weak lensing profile extrapolation (for radii $\gtrsim 1.5 r_{200}$ ) overestimates the profile by $\sim 20 \%-30 \%$. Cluster mass profiles may thus be steeper than NFW at these radii as Rines et al. (2012) suggest based on a much larger sample of clusters.

\section{CONCLUSION}

We compare cluster mass profiles derived with two fundamentally different methods. Weak lensing measures the projected surface mass density by analyzing small distortions of distant background galaxies (sources). The caustic technique is a kinematic technique based on the trumpet-like appearance of clusters in redshift space. Unlike a host of other mass estimation techniques, these two approaches are independent of equilibrium assumptions. In principle they can both be applied over a large radial range.

The 19 clusters in our sample span the mass range $\sim 10^{14}-10^{15} h^{-1} M_{\odot}$. The median ratio of the caustic and weak lensing mass profile is within the $68 \%$ confidence limits of the ratio between the true and caustic mass profiles derived from $N$-body simulations. At radii $\lesssim r_{200}$, the caustic approach overestimates the mass, a behavior expected as a result of a constant form factor. Near the virial radius $\left(\sim 1.3 r_{200}\right)$, the profiles agree to $\sim 30 \%$. At large radius, the extrapolated NFW fit to the weak lensing profiles appears to systematically overestimate the mass profile by $\sim 20 \%-30 \%$ in essential agreement with Rines et al. (2012). Direct measurement of weak lensing profiles based on more extensive imaging and extending to $3-4 r_{200}$ would provide an important test of the dynamical results.

Unlike weak lensing, the caustic technique is insensitive to superposed structures along the line of sight. We demonstrate by examining the system MS0906+11 that the impact of superposed structures (including other clusters) can as large as a factor of two. These results underscore the need for detailed simulations of potential biases produced by large-scale structure superposed within the weak lensing kernel.

Spectroscopic data are rarely used in combination with weak lensing. Our analysis suggests that weak lensing mass profiles could be improved by using a redshift survey to identify structures superposed along the line of sight and within the lensing kernel (see, for example, Coe et al. 2012). Furthermore, the agreement of masses derived near the virial radius suggests that a combination of a dense cluster redshift survey with weak lensing estimates could be the basis for a more powerful method of assessing the mass distribution at radii $\gtrsim r_{200}$ than either the caustic technique or weak lensing alone.

We thank Ian Dell'Antonio and Scott Kenyon for insightful discussions that helped us clarify issues in this paper. We thank the referee for lucid, incisive comments enabling us to clarify our weak lensing discussion. The Smithsonian Institution partially supports M.J.G.'s research. K.R. was funded by a Cottrell College Science Award from the Research Corporation. A.L.S. acknowledges a fellowship by the PRIN INAF09 "Toward an Italian Network for Computational Cosmology." A.D. and A.L.S. acknowledge partial support from the INFN grant PD51 and the PRIN-MIUR-2008 grant "Matter-antimatter asymmetry, dark matter and dark energy in the LHC Era."

Facilities: MMT(Hectospec) 


\section{REFERENCES}

Adelman-McCarthy, J. K., Agüeros, M. A., Allam, S. S., et al. 2006, ApJS, 162,38

Becker, M. R., \& Kravtsov, A. V. 2011, ApJ, 740, 25

Belsole, E., Pratt, G. W., Sauvageot, J.-L., \& Bourdin, H. 2004, A\&A, 415, 821

Biviano, A., \& Girardi, M. 2003, ApJ, 585, 205

Böhringer, H., Voges, W., Huchra, J. P., et al. 2000, ApJS, 129, 435

Broadhurst, T., Umetsu, K., Medezinski, E., Oguri, M., \& Rephaeli, Y. 2008, ApJL, 685, L9

Carlberg, R. G., Yee, H. K. C., Ellingson, E., et al. 1996, ApJ, 462, 32

Coe, D. 2010, arXiv:1005.0411

Coe, D., Umetsu, K., Zitrin, A., et al. 2012, ApJ, 757, 22

de Putter, R., \& White, M. 2005, NewA, 10, 676

Diaferio, A. 1999, MNRAS, 309, 610

Diaferio, A., \& Geller, M. J. 1997, ApJ, 481, 633

Diaferio, A., Geller, M. J., \& Rines, K. J. 2005, ApJL, 628, L97

Drinkwater, M. J., Gregg, M. D., \& Colless, M. 2001, ApJL, 548, L139

Duffy, A. R., Schaye, J., Kay, S. T., \& Dalla Vecchia, C. 2008, MNRAS, 390, L64

Ebeling, H., Edge, A. C., Bohringer, H., et al. 1998, MNRAS, 301, 881

Elvis, M., Plummer, D., Schachter, J., \& Fabbiano, G. 1992, ApJS, 80, 257

Faber, T., \& Visser, M. 2006, MNRAS, 372, 136

Fabricant, D., Fata, R., Roll, J., et al. 2005, PASP, 117, 1411

Forman, W., Bechtold, J., Blair, W., et al. 1981, ApJL, 243, L133

Geller, M. J., Diaferio, A., \& Kurtz, M. J. 1999, ApJL, 517, L23

Giles, P. A., Maughan, B. J., Birkinshaw, M., Worrall, D. M., \& Lancaster, K. 2012, MNRAS, 419, 503

Govoni, F., Markevitch, M., Vikhlinin, A., et al. 2004, ApJ, 605, 695

Hennawi, J. F., Dalal, N., Bode, P., \& Ostriker, J. P. 2007, ApJ, 654, 714

Hoekstra, H. 2001, A\&A, 370, 743

Hoekstra, H. 2003, MNRAS, 339, 1155

Hoekstra, H. 2007, MNRAS, 379, 317

Hoekstra, H., Hartlap, J., Hilbert, S., \& van Uitert, E. 2011, MNRAS, 412, 2095

Irgens, R. J., Lilje, P. B., Dahle, H., \& Maddox, S. J. 2002, ApJ, 579, 227

Kaiser, N. 1987, MNRAS, 227, 1

Kempner, J. C., Sarazin, C. L., \& Markevitch, M. 2003, ApJ, 593, 291

Kneib, J.-P., Hudelot, P., Ellis, R. S., et al. 2003, ApJ, 598, 804

Lam, T. Y., Nishimichi, T., Schmidt, F., \& Takada, M. 2012, PhRvL, 109, 051301

Lemze, D., Barkana, R., Broadhurst, T. J., \& Rephaeli, Y. 2008, MNRAS, 386, 1092
Lemze, D., Broadhurst, T., Rephaeli, Y., Barkana, R., \& Umetsu, K. 2009, ApJ, 701,1336

Lu, T., Gilbank, D. G., Balogh, M. L., et al. 2010, MNRAS, 403, 1787

Mahdavi, A., Geller, M. J., Böhringer, H., Kurtz, M. J., \& Ramella, M. 1999, ApJ, 518, 69

Markevitch, M., Ponman, T. J., Nulsen, P. E. J., et al. 2000, ApJ, 541, 542

Maughan, B. J., Jones, C., Forman, W., \& Van Speybroeck, L. 2008, ApJS, 174, 117

Meneghetti, M., Fedeli, C., Pace, F., Gottlöber, S., \& Yepes, G. 2010, A\&A, 519, A90

Merritt, D., Graham, A. W., Moore, B., Diemand, J., \& Terzić, B. 2006, AJ, 132,2685

Navarro, J. F., Frenk, C. S., \& White, S. D. M. 1997, ApJ, 490, 493

Navarro, J. F., Ludlow, A., Springel, V., et al. 2010, MNRAS, 402, 21

Oguri, M., \& Blandford, R. D. 2009, MNRAS, 392, 930

Oguri, M., Hennawi, J. F., Gladders, M. D., et al. 2009, ApJ, 699, 1038

Oguri, M., Takada, M., Okabe, N., \& Smith, G. P. 2010, MNRAS, 405, 2215

Okabe, N., Takada, M., Umetsu, K., Futamase, T., \& Smith, G. P. 2010, PASJ, 62,811

Okabe, N., \& Umetsu, K. 2008, PASJ, 60, 345

Postman, M., Coe, D., Benítez, N., et al. 2012, ApJS, 199, 25

Prada, F., Klypin, A. A., Cuesta, A. J., Betancort-Rijo, J. E., \& Primack, J. 2012, MNRAS, 423, 3018

Regös, E., \& Geller, M. J. 1989, AJ, 98, 755

Reisenegger, A., Quintana, H., Carrasco, E. R., \& Maze, J. 2000, AJ, 120, 523

Rines, K., \& Diaferio, A. 2006, AJ, 132, 1275

Rines, K., \& Diaferio, A. 2008, arXiv:0809.4015

Rines, K., \& Diaferio, A. 2010, AJ, 139, 580

Rines, K., Geller, M. J., Diaferio, A., \& Kurtz, M. J. 2012, arXiv:1209.3786

Rines, K., Geller, M. J., Diaferio, A., Mohr, J. J., \& Wegner, G. A. 2000, AJ, 120,2338

Rines, K., Geller, M. J., Diaferio, A., et al. 2002, AJ, 124, 1266

Rines, K., Geller, M. J., Kurtz, M. J., \& Diaferio, A. 2003, AJ, 126, 2152

Sereno, M., Jetzer, P., \& Lubini, M. 2010, MNRAS, 403, 2077

Serra, A. L., Diaferio, A., Murante, G., \& Borgani, S. 2011, MNRAS, 412,800

Serra, A. L., \& Domínguez Romero, M. J. L. 2011, MNRAS, 415, L74

Umetsu, K., Birkinshaw, M., Liu, G.-C., et al. 2009, ApJ, 694, 1643

Umetsu, K., Broadhurst, T., Zitrin, A., et al. 2011, ApJ, 738, 41

Umetsu, K., Medezinski, E., Broadhurst, T., et al. 2010, ApJ, 714, 1470

White, M., van Waerbeke, L., \& Mackey, J. 2002, ApJ, 575, 640 